\title{
Mepolizumab effectiveness and identification of super-responders in severe asthma
}

\author{
Erin S. Harvey ${ }^{1,2}$, David Langton ${ }^{3,4}$, Constance Katelaris ${ }^{5,6}$, Sean Stevens ${ }^{1}$, \\ Claude S. Farah (10 ${ }^{7}$, Andrew Gillman ${ }^{8}$, John Harrington ${ }^{2}$, Mark Hew ${ }^{8}$, \\ Vicky Kritikos ${ }^{9}$, Naghmeh Radhakrishna ${ }^{10}$, Philip Bardin ${ }^{11}$, Matthew Peters ${ }^{7}$, \\ Paul N. Reynolds ${ }^{12}$, John W. Upham ${ }^{13,14}$, Melissa Baraket ${ }^{15,16}$, Simon Bowler ${ }^{17}$, \\ Jeffrey Bowden ${ }^{18}$, Jimmy Chien ${ }^{19,20}$, Li Ping Chung ${ }^{21}$, Christopher Grainge ${ }^{2}$, \\ Christine Jenkins ${ }^{7,22}$, Gregory P. Katsoulotos ${ }^{23,24,25}$, Joy Lee ${ }^{26}$, Vanessa \\ M. McDonald ${ }^{1,2}$, Helen K. Reddel (10 ${ }^{9}$, Janet Rimmer ${ }^{25,27}$, Peter A.B. Wark (1) ${ }^{1,2}$ \\ and Peter G. Gibson ${ }^{1,2}$
}

@ERSpublications

In clinical practice, mepolizumab reduces the burden of severe eosinophilic asthma by reducing severe exacerbations and improving asthma control, quality of life and lung function. Super-responders have a T2 phenotype and few comorbidities. http://bit.ly/2Ulio4x

Cite this article as: Harvey ES, Langton D, Katelaris C, et al. Mepolizumab effectiveness and identification of super-responders in severe asthma. Eur Respir J 2020; 55: 1902420 [https://doi.org/10.1183/ 13993003.02420-2019].

ABSTRACT Severe asthma is a high-burden disease. Real-world data on mepolizumab in patients with severe eosinophilic asthma is needed to assess whether the data from randomised controlled trials are applicable in a broader population.

The Australian Mepolizumab Registry (AMR) was established with an aim to assess the use, effectiveness and safety of mepolizumab for severe eosinophilic asthma in Australia.

Patients ( $n=309$ ) with severe eosinophilic asthma (median age 60 years, 58\% female) commenced mepolizumab. They had poor symptom control (median Asthma Control Questionnaire (ACQ)-5 score of 3.4), frequent exacerbations (median three courses of oral corticosteroids (OCS) in the previous 12 months), and $47 \%$ required daily OCS. Median baseline peripheral blood eosinophil level was 590 cells $\mu \mathrm{L}^{-1}$. Comorbidities were common: allergic rhinitis $63 \%$, gastro-oesophageal reflux disease $52 \%$, obesity $46 \%$, nasal polyps $34 \%$.

Mepolizumab treatment reduced exacerbations requiring OCS compared with the previous year (annualised rate ratio 0.34 (95\% CI $0.29-0.41) ; \mathrm{p}<0.001$ ) and hospitalisations (rate ratio 0.46 (95\% CI 0.33-0.63); $\mathrm{p}<0.001$ ). Treatment improved symptom control (median ACQ-5 reduced by 2.0 at 6 months), quality of life and lung function. Higher blood eosinophil levels $(\mathrm{p}=0.003)$ and later age of asthma onset $(p=0.028)$ predicted a better ACQ-5 response to mepolizumab, whilst being male $(p=0.031)$ or having body mass index $\geqslant 30(\mathrm{p}=0.043)$ predicted a lesser response. Super-responders (upper $25 \%$ of ACQ-5 responders, $\mathrm{n}=61,24 \%$ ) had a higher $\mathrm{T} 2$ disease burden and fewer comorbidities at baseline.

Mepolizumab therapy effectively reduces the significant and long-standing disease burden faced by patients with severe eosinophilic asthma in a real-world setting.

This article has supplementary material available from erj.ersjournals.com

Received: 17 Dec 2019 | Accepted after revision: 7 Feb 2020

Copyright $\odot$ ERS 2020 


\section{Introduction}

Severe asthma is a high burden disease [1-5], for which oral corticosteroids (OCS) are frequently required. Novel therapies are needed to reduce the burden from symptoms, exacerbations and OCS toxicity. Mepolizumab, a humanised IgG1/ $\kappa$ anti-interleukin (IL)-5 monoclonal antibody, targets the eosinophilic subtype of severe asthma, and is highly effective in clinical trials [6-11].

However, the eligibility for registration trials of monoclonal antibody therapy in severe asthma is highly restricted, such that up to $90 \%$ of patients with severe asthma in a real-world setting may not be eligible for an efficacy trial [12]. This limits the generalisability of randomised controlled trial (RCT) data in severe asthma, and raises questions regarding the real-world effectiveness of monoclonal antibody therapy in severe asthma.

Real-world observational data from clinical registries can be used to answer these questions [13, 14]. However, real-world data for mepolizumab is limited [15-21]. The Australian Mepolizumab Registry (AMR) was established to address this knowledge gap. Mepolizumab was listed as a reimbursable medication on the Australian Pharmaceutical Benefits Scheme (PBS) in January 2017. Clinical features and blood eosinophil level are used to select patients for subsidised mepolizumab, and clinical response guides continuation of therapy (table 1).

We hypothesised that mepolizumab would result in positive clinical outcomes in a real-world severe eosinophilic asthma population. The aims of the AMR are to describe severe eosinophilic asthma in Australia, the effectiveness of mepolizumab, and adverse drug-related effects in this population.

\section{Methods}

\section{Study design}

The AMR is an investigator-initiated national, multi-centre observational post-marketing surveillance registry of patients treated with mepolizumab for severe uncontrolled eosinophilic asthma and enrolled through centres of the Australasian Severe Asthma Network (ASAN) [5]. Centres $(n=20)$ including hospital-based severe asthma, respiratory, immunology and allergy clinics and private respiratory practices, registered patients between January 2017 and April 2019. All sites received approval from the relevant Human Research Ethics Committee (supplementary material) and patients gave written informed consent prior to enrolment. The AMR was registered on the Australian New Zealand Clinical Trials Registry (ACTRN12618001497291)

Patients were assessed for eligibility (table 1) prior to initiation of mepolizumab and patients who commenced therapy were followed up prospectively. Baseline data were collected before commencement and patients received mepolizumab via their clinic or home service. Follow-up assessment occurred at 3 months post-commencement, and at 6 months and 12 months. Eligibility of patients to continue PBS-subsidised treatment was based on a reduction in ACQ-5 score of $\geqslant 0.5$ from baseline or reduction in maintenance OCS dose of $\geqslant 25 \%$ without deterioration in ACQ-5 (table 1).

Affiliations: ${ }^{1}$ Centre of Excellence in Severe Asthma and Priority Research Centre for Healthy Lungs, Faculty of Health, University of Newcastle, Newcastle, Australia. ${ }^{2}$ Dept of Respiratory and Sleep Medicine, John Hunter Hospital, Newcastle, Australia. ${ }^{3}$ Faculty of Medicine, Nursing and Health Sciences, Monash University, Clayton, Australia. ${ }^{4}$ Dept of Thoracic Medicine, Frankston Hospital, Frankston, Australia. ${ }^{5}$ School of Medicine, Western Sydney University, Campbelltown, Australia. ${ }^{6}$ Immunology and Allergy Unit, Campbelltown Hospital, Campbelltown, Australia. ${ }^{7}$ Dept of Thoracic Medicine, Concord Hospital, Concord, Australia. ${ }^{8}$ Allergy, Asthma and Clinical Immunology, Alfred Health, Melbourne, Australia. ${ }^{9}$ Dept of Respiratory and Sleep Medicine, Royal Prince Alfred Hospital, Camperdown, Australia. ${ }^{10}$ Respiratory Dept, St Vincent's Hospital, Melbourne, Australia. ${ }^{11}$ Lung and Sleep Medicine, Monash University and Medical Centre, Clayton, Australia. ${ }^{12}$ Lung Research, Hanson Institute and Dept of Thoracic Medicine, Royal Adelaide Hospital, Adelaide, Australia. ${ }^{13}$ Dept of Respiratory Medicine, Princess Alexandra Hospital, Woolloongabba, Australia. ${ }^{14}$ The University of Queensland Diamantina Institute, Woolloongabba, Australia. ${ }^{15}$ South Western Sydney Clinical School, University of New South Wales, Sydney, Australia. ${ }^{16}$ Ingham Institute for Applied Medical Research, Sydney, Australia. ${ }^{17}$ Dept of Respiratory Medicine, Mater Hospital Brisbane, South Brisbane, Australia. ${ }^{18}$ Respiratory and Sleep Services, Flinders Medical Centre and Flinders University, Bedford Park, Australia. ${ }^{19}$ Dept of Sleep and Respiratory Medicine, Westmead Hospital, Westmead, Australia. ${ }^{20}$ School of Medicine, The University of Sydney, Sydney, Australia. ${ }^{21}$ Dept of Respiratory Medicine, Fiona Stanley Hospital, Murdoch, Australia. ${ }^{22}$ Concord Clinical School University of Sydney, Concord, Australia. ${ }^{23}$ St George Specialist Centre, Kogarah, Australia. ${ }^{24}$ St George and Sutherland Clinical School, University of New South Wales, Sydney, Australia.

${ }^{25}$ Woolcock Institute of Medical Research, Glebe, Australia. ${ }^{26}$ Austin Health and Monash University, Melbourne, Australia. ${ }^{27}$ St Vincent's Clinic, Darlinghurst, Australia.

Correspondence: Peter G. Gibson, Hunter Medical Research Institute, Lot 1 Kookaburra Circuit, New Lambton Heights NSW 2305, Australia. E-mail: peter.gibsonahealth.nsw.gov.au 
TABLE 1 Inclusion, continuation and exclusion criteria for Australian Mepolizumab Registry participation

\section{Inclusion criteria}

Able to provide informed written consent

Age $\geqslant 12$ years $\#$

Asthma diagnosis confirmed by doctor or hospital and documented for $\geqslant 1$ year $\#$

Confirmed variable airflow obstruction lone or more of the following) ${ }^{\#}$ :

- $\mathrm{FEV}_{1}$ reversibility $\geqslant 12 \%$ and $\geqslant 200 \mathrm{~mL}$ at baseline within $30 \mathrm{~min}$ after administration of salbutamol $(200-400 \mu \mathrm{g})$, OR

- Airway hyper-responsiveness defined as $>20 \%$ decline in $\mathrm{FEV}_{1}$ during a direct bronchial provocation test or $>15 \%$ decline during an indirect test, OR

- Peak expiratory flow variability of $>15 \%$ between the two highest and two lowest peak expiratory flow rates during 14 days

Optimised asthma management skills (formal assessment of and adherence to correct inhaler technique, documented in medical records)

$\mathrm{FEV}_{1} \leqslant 80 \%$ predicted documented on one or more occasions in the previous 12 months ${ }^{\#}$

Optimised asthma therapy (unless contraindicated or not tolerated) ${ }^{\#}$ :

- $\geqslant 12$ months maximal inhaled therapy with adherence and correct technique documented, including:

o High-dose inhaled corticosteroid

$\circ$ Long-acting $\beta_{2}$ agonist therapy

AND

- Treatment with oral corticosteroids (OCS) (daily OCS for at least 6 weeks OR a cumulative dose of $\geqslant 500 \mathrm{mg}$ prednisolone equivalent in the previous 12 months)

Uncontrolled asthma, as defined by

- Asthma Control Questionnaire (ACQ-5) score $\geqslant 2$ in the previous month AND

- One of the following experienced in the previous year:

$0 \geqslant 1$ admission to hospital for a severe asthma exacerbation, OR

$0 \geqslant 1$ severe asthma exacerbation requiring documented use of systemic corticosteroids (OCS

initiated or increased for at least 3 days, or parenteral corticosteroids) prescribed/supervised by a physician.

Eosinophilic asthma as determined from peripheral blood eosinophil count $\geqslant 300$ cells $\mu \mathrm{L}^{-1}$ in the previous 12 months ${ }^{\#}$

\section{Continuation criteria for government-subsidised treatment with mepolizumab}

Demonstrated or sustained an adequate response to mepolizumab treatment as evidenced by: "

- A reduction in the Asthma Control Questionnaire (ACQ)-5 score of at least 0.5 from baseline OR

- A reduction in the maintenance oral dose of corticosteroid by at least $25 \%$ from baseline AND

No deterioration in the ACQ-5 from baseline

\section{Exclusion criteria}

Not receiving mepolizumab treatment in combination with or within 6 months of PBS subsidised omalizumab treatment $\#$,

People highly dependent on medical care

Cognitive impairment preventing completion of data collection forms

Australian Commonwealth Government Pharmaceutical Benefits Scheme (PBS) criteria for \#initiation of a subsidised mepolizumab cycle and "continuation of PBS-subsidised mepolizumab after 26-30 weeks of treatment (1st continuation assessment), 18-22 weeks (subsequent continuation assessments).

\section{Patients}

Patients $\geqslant 12$ years $(n=339)$ with severe eosinophilic asthma were enrolled and 309 patients commenced PBS-subsidised mepolizumab. Patient demographics, medical history, asthma diagnosis and history, exacerbation history, medication use and healthcare resource utilisation were recorded. Patients completed the five-item Asthma Control Questionnaire (ACQ)-5 [22], Asthma Control Test (ACT) [23] and standardised Asthma Quality of Life Questionnaire (AQLQ(S)) [24]. Spirometry (within month prior to starting mepolizumab), fraction of exhaled nitric oxide $\left(F_{\mathrm{eNO}}\right)$, atopic status (skin prick test (SPT)/radioallergosorbent test/ImmunoCAP assay or previous omalizumab treatment), full blood count (including blood eosinophil level) and total serum immunoglobulin (Ig)E concentration (within 12 months prior) were recorded. Outcome data were collected at 3 months, 6 months and 12 months post-commencement of mepolizumab, and included frequency of severe exacerbations, change in ACQ-5, health-related quality of life (HRQoL)((AQLQ 
(S)), medication use, lung function and blood eosinophil levels. Patients who continued PBS-subsidised treatment after 6 months were identified as responders and further classified as "super-responders" (top 25\% of ACQ-5 responses from baseline or well-controlled asthma symptoms (ACQ-5<1.0)) [25].

\section{Database management and quality control}

Data were collected and managed using REDCap electronic data capture tools hosted at Hunter Medical Research Institute, Newcastle, Australia [26]. The AMR REDCap template was adapted from data collection platforms of the ASAN Severe Asthma Web-based Database [5] and the Alfred Difficult Asthma Service Melbourne, Australia [27].

\section{Statistical analysis}

Statistical analyses were performed using Stata14.2 (StataCorp, College Station, TX, USA); results are reported as mean $\pm \mathrm{SD}$ for normally distributed data and median (interquartile range) for non-normally distributed data. Comparisons were performed using Chi-squared or Fisher's exact test for categorical data and Student's t-test or Wilcoxon rank-sum test for continuous data. The annualised rate of exacerbations for the study period was analysed using a negative binomial generalised estimating equation incorporating repeated measures across patients, producing population-averaged estimates. The within-group correlation structure was specified as exchangeable and a logarithm of time was included as an offset variable. From this, the rate ratio comparing exacerbations prior to and post-commencement of mepolizumab treatment was calculated, as well as the estimated rate per year and associated 95\% confidence intervals. Baseline predictors of ACQ-5 response were evaluated using multiple linear regression. Variables for the multivariate models were chosen if the p-value was $<0.2$ in the linear model (supplementary material). Results were considered significant when $\mathrm{p}<0.05$.

\section{Results}

Patients ( $n=339)$ were enrolled in AMR and $n=309$ confirmed eligible patients commenced PBS-subsidised mepolizumab (figure 1). After 6-months of treatment, there were 260 (86\%) out of 302 mepolizumab responders (according to PBS-continuation criteria, table 1), after excluding patients awaiting assessment $(\mathrm{n}=7)$. Of the responders, $197(93 \%)$ out of 211 were still responders at the 12 -month assessment, after excluding patients awaiting assessment $(n=49)$.

\section{Baseline characteristics}

Patients had a median age of 60 years and $58 \%$ were female (table 2). Patients had poor symptom control, median (IQR) ACQ-5 score of 3.4 (3.0-4.2), and 28\% had been hospitalised for an asthma exacerbation in the previous year. Comorbidities were common (supplementary table S1). At baseline, $47 \%$ of patients were taking prescribed maintenance OCS at a median (IQR) daily dose of 10 (5-12.5) $\mathrm{mg} \cdot \mathrm{day}^{-1}$ prednisolone equivalent. Patients were using median (IQR) 4 (3-5) respiratory medications for their severe asthma therapy. In addition to inhaled corticosteroid (ICS) and long-acting $\beta$-agonist (LABA), long-acting muscarinic antagonist (LAMA) was used by $53 \%$, montelukast by $12 \%$, theophylline by $6 \%$, antifungal azoles by $3 \%$, methotrexate by $4 \%$ and low-dose macrolides by $9 \% .16 \%$ of patients had received prior treatment with omalizumab, $3 \%$ had received prior anti-IL5 monoclonal antibody therapy, and $4 \%$ had been treated with bronchial thermoplasty.

\section{Response to mepolizumab}

Patients received mepolizumab treatment for 352 (223-377) days. Significant and sustained biological and clinical responses to mepolizumab were reported over the 12-month period (figure 2). Blood eosinophil levels reduced from median (IQR) 590 (400-830) cells. $\mu \mathrm{L}^{-1}$ at baseline to 100 (40-100) cells. $\mu \mathrm{L}^{-1}$ $(\mathrm{p}<0.001)$ at the 3-month assessment, and remained at this level at the 12-month assessment (figure 2a).

Patients reported a significant improvement in symptom control (ACQ-5 reduction) (ACQ-5 median (IQR) $3.4(3.0-4.2)$ at baseline versus $1.6(0.8-2.6)$ at the 3 -month assessment, $\mathrm{p}<0.001)$. The improvement in ACQ-5 was maintained at subsequent assessments (figure 2b). Patients reported significant and increasing improvements in HRQoL (figure 2c) and lung function improved (figure 2d).

There was a significant reduction in the annualised rates of severe asthma exacerbations in comparison to the 12 months prior to treatment (figure 3) $(n=306)$. The rate of exacerbation (per person-year) requiring OCS was reduced by $66 \%$ (95\% CI 59-71), p $<0.001$, requiring hospitalisation was reduced by $54 \%(95 \%$ CI 37-67), $\mathrm{p}<0.001$, and emergency room visitation by $56 \%$ (95\% CI $25-74), \mathrm{p}=0.003$. The reduction in the rate of exacerbations was greatest for exacerbations resulting in unscheduled doctor visits, with a reduction of $83 \%$ (95\% CI $72-89$ ), $\mathrm{p}<0.001$.

Following mepolizumab commencement, the proportion of patients requiring maintenance OCS was reduced from $47 \%$ at baseline to $30 \%$ at the 6 -month assessment $(\mathrm{p}<0.001)$, with the daily dose reduced from median (IQR) $10(5.0-12.5)$ to $7.5(5.0-10.0) \mathrm{mg} \cdot \mathrm{day}^{-1}$ prednisolone equivalent $(\mathrm{p}=0.001)$ in patients requiring OCS. 


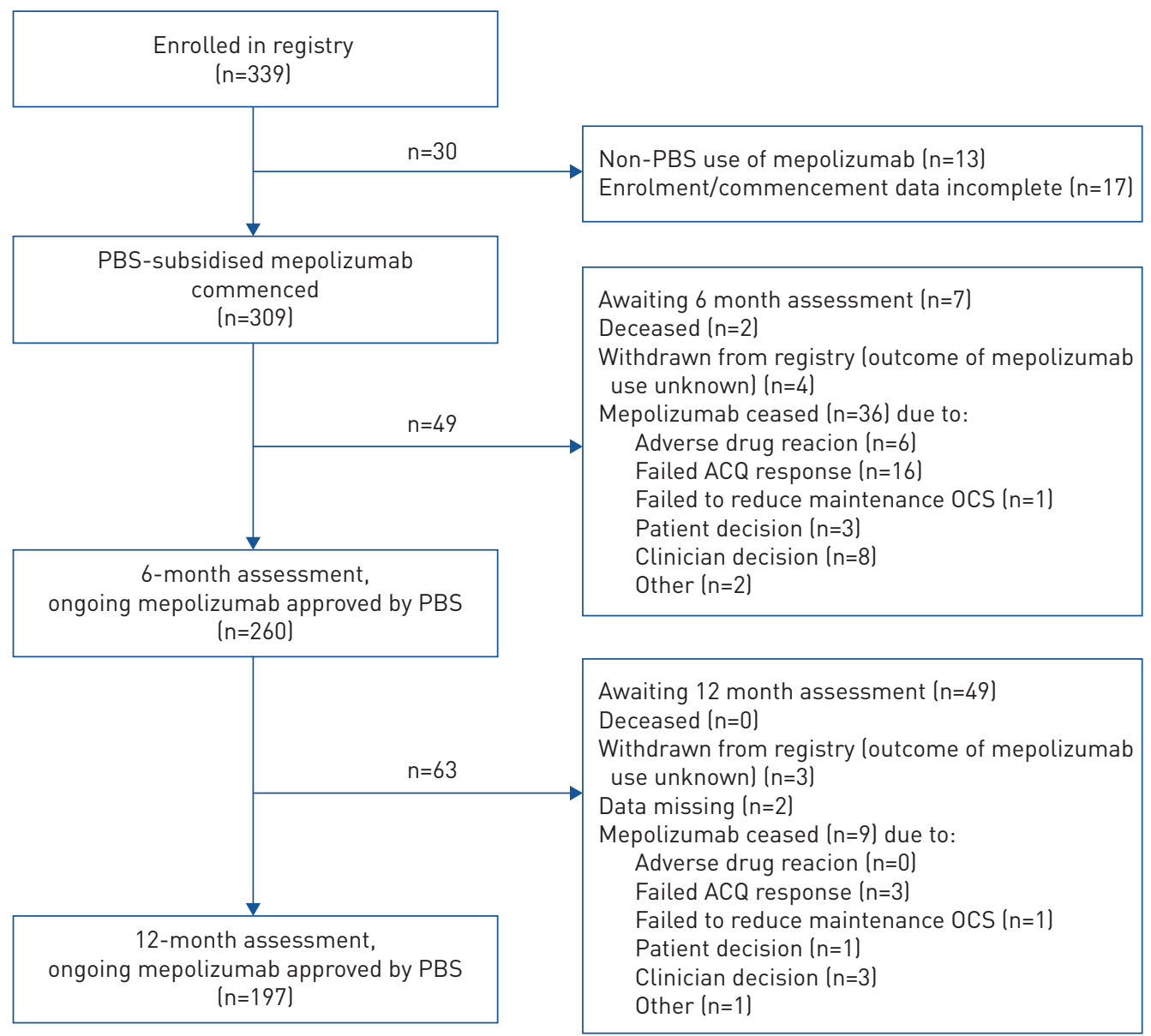

FIGURE 1 Flow of patients enrolled in the Australian Mepolizumab Registry with ongoing mepolizumab therapy approved. Patients were enrolled between January 2017 and April 2019. The initial 12 months of treatment, including the outcome of PBS assessment for continuation of ongoing mepolizumab therapy, and reasons for mepolizumab cessation are shown. ACQ: Asthma Control Questionnaire; OCS: oral corticosteroid; PBS: Pharmaceutical Benefits Scheme of the Australian Commonwealth Government, which subsidises mepolizumab for patients with severe eosinophilic asthma who meet certain criteria (see table 1).

\section{Cessation of mepolizumab}

Mepolizumab was ceased by 45 (14.6\%) out of 309 patients during the 12-month period (figure 1 and table 3). The most common reasons for cessation were failed ACQ response and clinician decision.

\section{Safety of mepolizumab}

Mepolizumab administration data were available for 2374 mepolizumab administration events (table 4). An adverse drug reaction was reported for $18.8 \%$ of patients. The most-commonly reported reactions were headache (9.9\% of patients). No anaphylaxis events were reported. Mepolizumab treatment was ceased by six patients due to adverse drug reactions.

Of 25 recorded severe adverse effects (SAEs) (supplementary table S2), two were fatal (cause of death recorded: acute myocardial infarction and ischaemic heart disease). None of the SAEs had suspected causality to mepolizumab. Treatment was temporarily adjusted or interrupted following four SAEs and permanently discontinued following one (metastatic brain lesion).

\section{Response to mepolizumab by baseline blood eosinophil level}

Patients with baseline blood eosinophil levels $>600$ cells $\mu \mathrm{L}^{-1}$ (Eo-high patients, $42 \%$ ), reported a greater improvement in ACQ-5 score relative to baseline at the 3-month assessment compared to patients with blood eosinophils $\leqslant 600$ cells $\mu \mathrm{L}^{-1}$ (Eo-low) (mean \pm SD ACQ-5 improvement of $-2.0 \pm 1.3$ versus $-1.6 \pm 1.2$; $\mathrm{p}=0.013)$. The magnitude of response remained significantly greater in the Eo-high patients at 6-months $(\mathrm{p}=0.003)$ and 12-months $(\mathrm{p}=0.047)$ (supplementary table S3).

Eo-high patients reported a significantly greater improvement in AQLQ(S) score at 3-months (mean \pm SDAQLQ (S) improvement of $1.4 \pm 1.2$ versus $1.0 \pm 1.1 ; \mathrm{p}=0.019$ ) and 6-months (mean \pm sDAQLQ(S) $1.6 \pm 1.3$ versus $1.1 \pm 1.1$; 
TABLE 2 Characteristics of patients prior to commencement of mepolizumab

\section{Demographics}

\begin{tabular}{|c|c|}
\hline Patients n & 309 \\
\hline Age years & 59.55 (49.99-68.28) \\
\hline Male sex & $131(42.4)$ \\
\hline \multicolumn{2}{|l|}{ Race } \\
\hline White & 252 (87.5) \\
\hline Asian & $21(7.3)$ \\
\hline Pacific Islander & $4(1.4)$ \\
\hline Other & $10(3.5)$ \\
\hline ATSI & $1(0.3)$ \\
\hline \multicolumn{2}{|l|}{ Smoking } \\
\hline Never smoker & $186(61.2)$ \\
\hline Current smoker & $2(0.6)$ \\
\hline Ex-smoker & $116(38.2)$ \\
\hline Pack-years (ex-/current smokers) (n=112) & $15.00(5.00-30.00)$ \\
\hline Body mass index $\mathrm{kg} \cdot \mathrm{m}^{-2}(\mathrm{n}=297)$ & $29.45(25.30-34.46)$ \\
\hline Obese (body mass index $\geqslant 30 \mathrm{~kg} \cdot \mathrm{m}^{-2}$ ) & $136(45.8)$ \\
\hline Atopy ${ }^{\#}(n=221)$ & $156(70.6)$ \\
\hline \multicolumn{2}{|l|}{ Asthma history } \\
\hline Age of asthma symptoms onset years ( $n=274)$ & $26.00(8.00-44.00)$ \\
\hline Asthma duration years & $27.52(13.30-46.08)$ \\
\hline \multicolumn{2}{|l|}{ Exacerbation history (past year) } \\
\hline Course of OCS for exacerbation & $295(95.5)$ \\
\hline Number of OCS courses ( $n=287$ ) & $3.00(2.00-6.00)$ \\
\hline Hospital admission & $85(27.5)$ \\
\hline Number of hospital admissions ( $n=85$ ) & $1.00(1.00-2.00)$ \\
\hline Intensive care unit admission & $6(1.9)$ \\
\hline Unscheduled doctor/LMO visit & $82(26.5)$ \\
\hline Number of unscheduled doctor/LMO visits ( $\mathrm{n}=81$ ) & $4.00(2.00-6.00)$ \\
\hline \multicolumn{2}{|l|}{ Lung function } \\
\hline \multicolumn{2}{|l|}{ Pre-Bronchodilator spirometry } \\
\hline $\mathrm{FEV}_{1} \%$ predicted $(\mathrm{n}=233)$ & $56.90 \pm 17.78$ \\
\hline FVC \% predicted $(n=232)$ & $78.53 \pm 16.70$ \\
\hline $\mathrm{FEV}_{1} / \mathrm{FVC}(\mathrm{n}=232)$ & $0.57 \pm 0.13$ \\
\hline \multicolumn{2}{|l|}{ Post-Bronchodilator spirometry } \\
\hline $\mathrm{FEV}_{1} \%$ predicted $(\mathrm{n}=211)$ & $62.65 \pm 19.03$ \\
\hline FVC $\%$ predicted $(n=211)$ & $83.57 \pm 16.56$ \\
\hline $\mathrm{FEV}_{1} / \mathrm{FVC}(\mathrm{n}=211)$ & $0.59 \pm 0.14$ \\
\hline $\mathrm{BDR}\left(\geqslant 12 \% \mathrm{FEV}_{1}\right.$ change) & $70(34.3)$ \\
\hline \multicolumn{2}{|l|}{ Asthma control } \\
\hline Asthma Control Questionnaire-5 score $(n=306)$ & $3.4(3.0-4.2)$ \\
\hline Asthma Control Test total score $(n=232)$ & $11(8.00-15.00)$ \\
\hline \multicolumn{2}{|l|}{ Asthma symptoms in past week } \\
\hline Number of nights woken due to asthma symptoms (n=219) & $3.00(1.00-7.00)$ \\
\hline Number of mornings with asthma symptoms on awakening $(n=219)$ & $6.00(3.00-7.00)$ \\
\hline Number of days with activity limitation due to asthma (n=218) & $7.00(3.00-7.00)$ \\
\hline Number of days of reliever use $(n=216)$ & $7.00(4.00,7.00)$ \\
\hline \multicolumn{2}{|l|}{ Biomarkers } \\
\hline Peripheral blood eosinophils $\mu \mathrm{L}^{-1}(\mathrm{n}=303)$ & $590(400-830)$ \\
\hline $\lg E \mathrm{IU} \cdot \mathrm{mL}^{-1}(\mathrm{n}=202)$ & $137.5(53.0-479.0)$ \\
\hline$F_{\mathrm{eNO}} \mathrm{ppb}(\mathrm{n}=147)$ & $35(20-61)$ \\
\hline
\end{tabular}

Data presented as mean \pm SD, median (IQR) or $\mathrm{n}(\%)$, unless otherwise stated. ATSI: Aboriginal or Torres Strait Islander; OCS: oral corticosteroids; LMO: local medical officer; FEV ${ }_{1}$ : forced expiratory volume in $1 \mathrm{~s}$; FVC: forced vital capacity; BDR: bronchodilator reversibility; Ig: immunoglobulin; $F_{\mathrm{eNO}}$ : fraction of exhaled nitric oxide. \#: Atopy positive classified by positive skin prick test or radioallergosorbent test/lmmunoCAP and/or previous omalizumab treatment.

$\mathrm{p}=0.026$ ). There was greater improvement in lung function in Eo-high patients at 12-months post-mepolizumab commencement (median (IQR) forced expiratory volume in $1 \mathrm{~s}\left(\mathrm{FEV}_{1}\right) \%$ predicted improvement of $8.0(-0.9-16.3)$ versus $3.2(-1.7-8.8) \%$; $=0.032)$ compared with patients with blood eosinophils $\leqslant 600$ cells $\mu \mathrm{L}^{-1}$. 
Baseline

a)

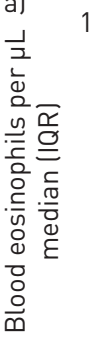

3-months

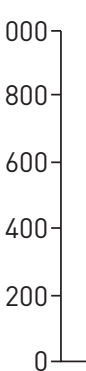

Baseline

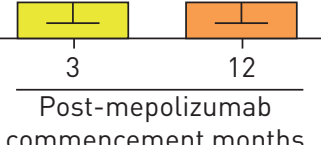

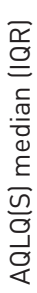

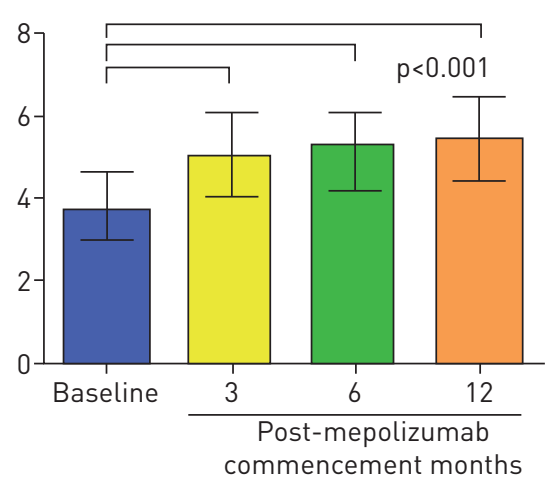

6-months

12-months
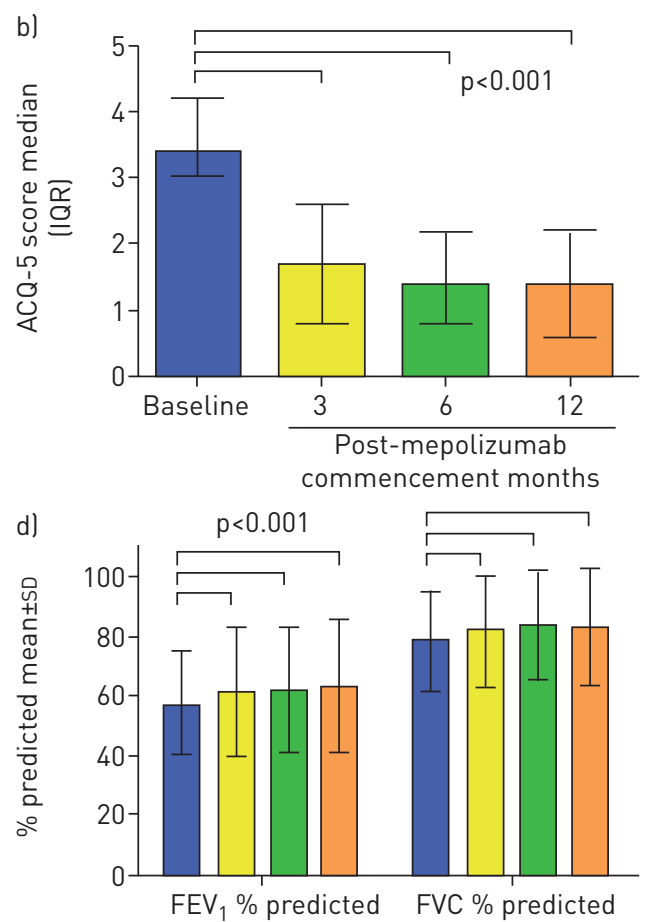

FIGURE 2 Clinical and biological response to mepolizumab (a) median (IQR) peripheral blood eosinophil levels during the 12 months prior to commencement of mepolizumab and at follow-up assessments, (b) median (IQR) Asthma Control Questionnaire (ACQ)-5 score at baseline and follow-up assessments, (c) median (IQR) standardised Asthma Quality of Life Questionnaire (AQLQ(S)) score at baseline and follow-up assessments, (d) mean \pm SD pre-bronchodilator percent of predicted forced expiratory volume in $1 \mathrm{~s}\left(\mathrm{FEV}_{1}\right)$ and forced vital capacity (FVC) at baseline and follow-up assessments. p-values represent difference relative to baseline using generalised estimating equations.

\section{Mepolizumab response predictors}

Patients with late onset asthma (onset $\geqslant 40$ years of age, $\mathrm{n}=86,31 \%$ ) were predominantly male with greater smoking exposure. They had a median (IQR) asthma duration of 12.3 (4.9-18.8) years compared with 37.9 (24.3-51.9) years, $\mathrm{p}<0.001$, in early onset asthma ( $<40$ years). Clinical responses to mepolizumab were similar in both the early and late age of onset categories (supplementary tables S4 and S5), and in non-atopic and atopic patients with eosinophilic asthma (supplementary tables S4 and S6).

In multivariate regression analysis models, male sex $(\mathrm{p}=0.031)$ and body mass index $(\mathrm{BMI}) \geqslant 30 \mathrm{~kg} \cdot \mathrm{m}^{-2}$ $(\mathrm{p}=0.043)$ predicted reduced ACQ-5 response, whilst higher baseline blood eosinophil levels $(\mathrm{p}=0.003)$ and later age of asthma onset $(\mathrm{p}=0.028)$ predicted greater improvement in ACQ-5 response. Age did not significantly predict ACQ-5 response in the multivariate model.

Patients identified as responders had similar baseline ACQ-5 scores, higher blood eosinophil levels and were less likely to be using maintenance OCS, compared to treatment-failures (supplementary table S7 and S8).

\section{Mepolizumab super-responders}

Mepolizumab treatment resulted in a median (IQR) reduction in ACQ-5 score of $-2.0(-1.4-3.0)$ in responders, with $79(31 \%)$ patients achieving well-controlled symptoms (ACQ-5 $<1.0)$. Clinicians recognise some patients who have an exceptionally good response, termed "super-responders". Super-responders were defined as those in the top 25\% of ACQ responses (61 out of 252 PBS-defined responders), or those who achieved well-controlled asthma after 6-months of treatment (79 out of 252) (supplementary table S8). The ACQ super-responders had a median (IQR) ACQ-5 score reduction of $-3.4(-3.2-4.0)$ units, compared with $-1.0(-0.6-1.2)$ in the bottom $25 \%$ of responders (figure 4a). Patients who achieved well-controlled asthma had a median ACQ-5 reduction of $-3.0(-2.4-3.6)$, compared with $-1.4(-1.0-2.0)$ in those whose symptoms remained uncontrolled (ACQ-5 $>1.5$ ) at 6-months. 
Rate ratio 0.34

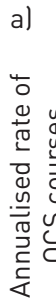

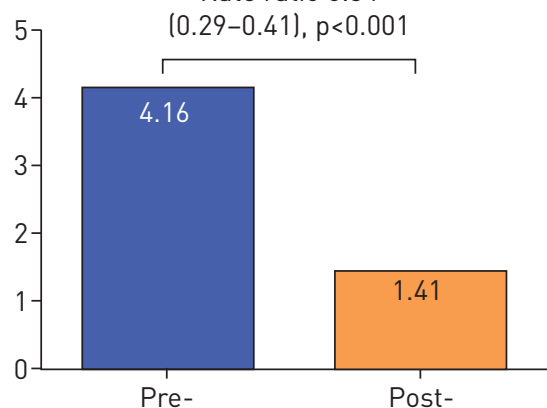

Mepolizumab treatment

Rate ratio 0.44

(0.26-0.75), $p<0.01$

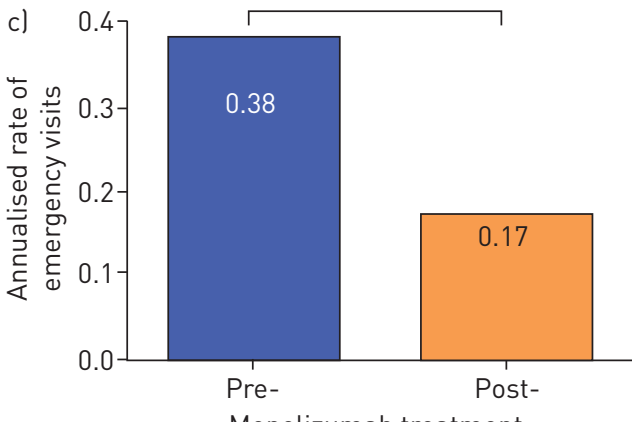

Rate ratio 0.46

(0.33-0.63), $p<0.001$

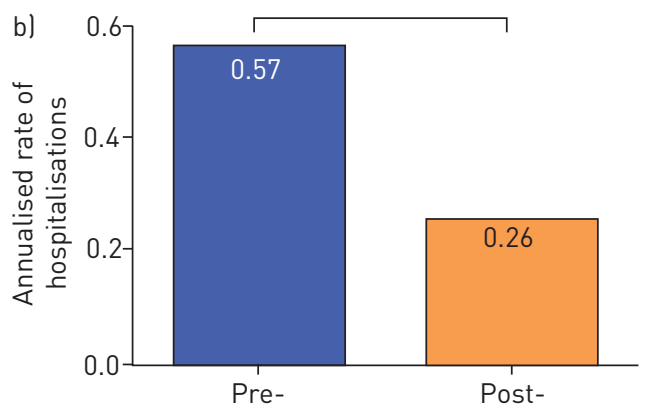

Mepolizumab treatment

Rate ratio 0.17

(0.11-0.28), $p<0.001$

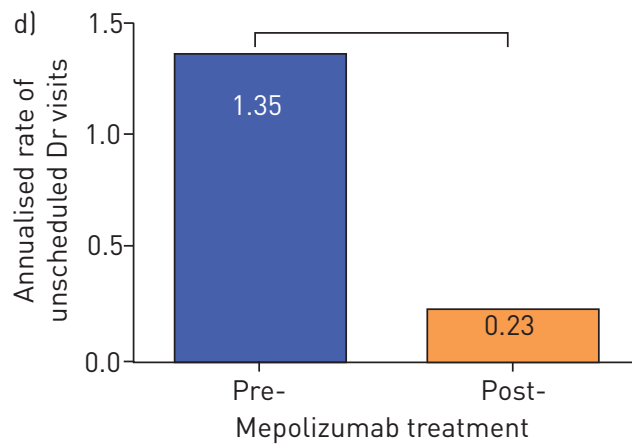

FIGURE 3 Severe asthma exacerbations during mepolizumab treatment and 12 months prior to treatment. Exacerbations per person-year and incidence rate ratio. Point estimate of annualised asthma exacerbation rate with $95 \%$ confidence intervals is shown for exacerbations resulting in a) oral corticosteroid treatment, b) hospitalisation, c) emergency room visit and d) unscheduled doctor visits. OCS: oral corticosteroids.

ACQ super-responders were predominantly female (67\% versus $43 \%$; $\mathrm{p}=0.005)$, had a lower baseline BMI (median $27.8(23.8-31.8)$ versus $\left.31.0(26.4-35.7) \mathrm{kg} \cdot \mathrm{m}^{-2} ; \mathrm{p}=0.006\right)$, tended to never have smoked $(70 \%$ versus 56\%; $\mathrm{p}=0.094)$ and had a shorter duration of asthma (median 23.6 (10.7-37.9) versus 34.1 (17.943.6) years; $\mathrm{p}=0.037$ ). They had higher baseline blood eosinophils (median 700 (430-1010) versus TABLE 3 Reasons for cessation of mepolizumab, and final pre-cessation level of asthma
symptom control

Reason for cessation of
mepolizumab $\quad \begin{gathered}\text { Number ceased } \\ \text { by reason } n\end{gathered}$

Final ACQ-5 score, pre-cessation median (IQR); (number of patients for whom final ACQ-5 data were available)

\begin{tabular}{lc}
\hline Total patients & 309 \\
Patient deceased & 2 \\
Adverse drug reaction & 6 \\
Failed ACQ response & 19 \\
Failed to reduce & 2 \\
$\quad$ maintenance OCS & \\
Patient decision & 4 \\
Clinician decision & 11 \\
Other & 3 \\
Total (not including & 45 \\
$\quad$ deceased patients) & \\
Total (including deceased & 47 \\
$\quad$ patients)
\end{tabular}

$1.40 ;(n=1)$

$1.8(1.2-3.0) ;(n=3)$

$3.6(2.8-4.2) ;(n=18)$

$2.80 ;(n=1)$

$2.1(1.5,2.9) ;(n=4)$

$2.3(2.0-2.6) ;(n=6)$

$2.00 ;(n=1)$

$2.8(2.0-3.6) ;(n=34)$

$2.7(2.0-3.6) ;(n=34)$

Data presented as median (IQR), unless otherwise stated. ACQ: Asthma Control Questionnaire; OCS: oral corticosteroids. 
TABLE 4 Adverse drug-related reactions in patients treated with mepolizumab

\begin{tabular}{|c|c|}
\hline Patients n & 309 \\
\hline Total mepolizumab administration events $\mathrm{n}^{\#}$ & $2374(n=308)$ \\
\hline Adverse drug reaction reported ${ }^{\text {I }}$ & $57 / 304(18.8 \%)$ \\
\hline \multicolumn{2}{|l|}{ Reaction type } \\
\hline Anaphylaxis & $0 / 304(0 \%)$ \\
\hline Urticaria & $7(2.3 \%)$ \\
\hline Angioedema & $1(0.3 \%)$ \\
\hline Rash & $10(3.3 \%)$ \\
\hline Bronchospasm & $0(0 \%)$ \\
\hline Hypotension & $0(0 \%)$ \\
\hline Headache & $30(9.9 \%)$ \\
\hline Local site reaction & $10(3.3 \%)$ \\
\hline Other & $30(9.9 \%)$ \\
\hline Mepolizumab ceased due to adverse drug reaction & $6 / 309(1.9 \%)$ \\
\hline \multicolumn{2}{|l|}{ Reaction } \\
\hline Severe headache & 2 \\
\hline Persistent eczematous reaction involving face, trunk and upper limbs & 1 \\
\hline Arthralgia & 1 \\
\hline Pruritus & 1 \\
\hline Middle back pain & 1 \\
\hline
\end{tabular}

Data are presented as $\mathrm{n}(\%), \mathrm{n} / \mathrm{N}(\%)$ or $\mathrm{n}$, unless otherwise stated. ${ }^{\#}$ : Administration event missing for one patient; ": adverse drug reaction data missing for five patients.

535 (400-805) cells $\left.\mu \mathrm{L}^{-1} ; \mathrm{p}=0.043\right)$ and $F_{\mathrm{eNO}}(41$ (21-63) versus 23 (15-41) ppb; $\mathrm{p}=0.026$ ) (figure 4b) and a higher baseline ACQ-5 (median $4.2(3.6-4.8)$ versus $3.0(2.6-3.6) ; \mathrm{p}<0.001)$. They were less likely to be taking maintenance OCS at baseline ( $36 \%$ versus $56 \%$; $=0.025)$. ACQ super-responders were more likely to have a diagnosis of nasal polyps ( $48 \%$ versus $30 \%$; $=0.035$ ), whilst gastro-oesophageal reflux disease (GORD) (35\% versus 52\%; $\mathrm{p}=0.046$ ), obstructive sleep apnoea (OSA) ( $16 \%$ versus $36 \%$; $\mathrm{p}=0.010$ ), psychiatric disorders ( $21 \%$ versus $46 \% ; \mathrm{p}=0.003$ ) and cardiovascular disease (CVD) (12\% versus $34 \%$; $\mathrm{p}=0.004$ ) were less common (figure $4 \mathrm{c}$ ).

Patients who achieved well-controlled symptoms after 6-months of treatment (ACQ-5 <1.0, n=79) were compared to those with ACQ-5>1.5 (uncontrolled, $\mathrm{n}=111$ ). Patients who achieved control were more likely to be never smokers (78\% versus 55\%; $\mathrm{p}=0.002)$ and had a lower baseline BMI (median 27.5 (23.8-30.5) versus $\left.31.8(27.1-36.6) \mathrm{kg} \cdot \mathrm{m}^{-2} ; \mathrm{p}<0.001\right)$. They had significantly less symptoms at baseline; (median ACQ-5 score $3.4(2.8-4.0)$ versus $3.6(3.2-4.4) ; \mathrm{p}<0.001)$, and had required less OCS treatment for exacerbations in the previous year $(3(2-5)$ versus $4(2-7) ; \mathrm{p}=0.046)$. They had higher $F_{\text {eNO }}$ levels (median 50.8 (29.0-94.0)
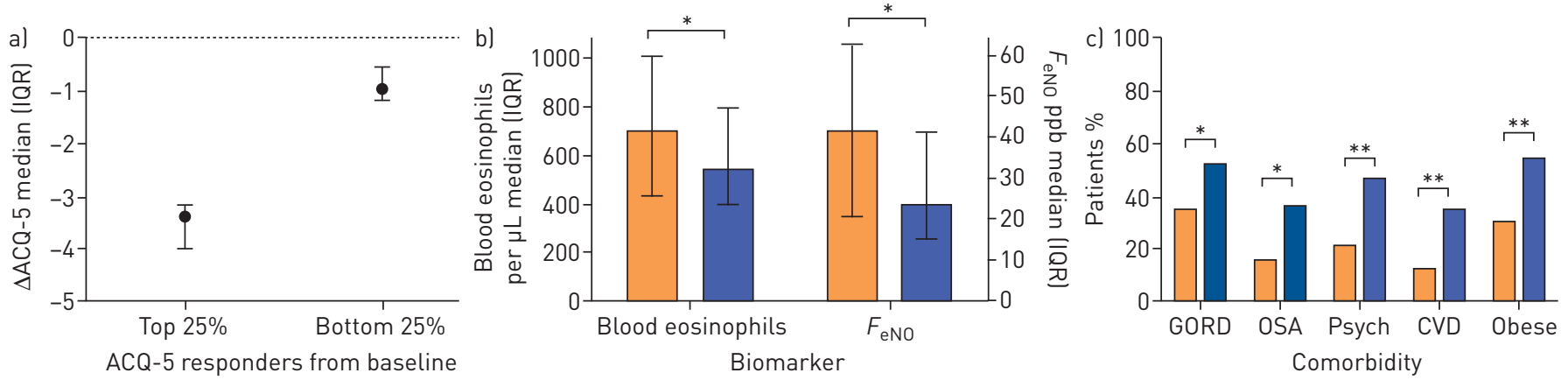

FIGURE 4 Response, biomarker and comorbidity profiles of mepolizumab super-responders. Asthma Control Questionnaire (ACQ)-5 response at the 6-month assessment relative to baseline was used to identify and compare the top $25 \%$ of $A C Q$ responders (super-responders) and the

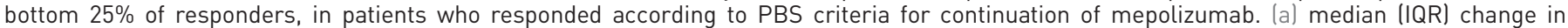

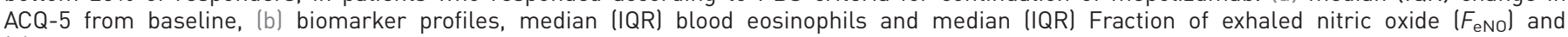
(c) comorbidity profiles. ACQ: Asthma Control Questionnaire; CVD: cardiovascular disease; GORD: gastro-oesophageal reflux disease; Psych: psychiatric disorder (anxiety/depression/other); PBS: Pharmaceutical Benefits Scheme of the Australian Commonwealth Government, which

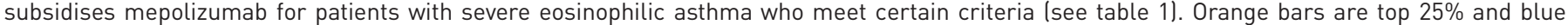
bars are bottom $25 \%{ }^{*}: p<0.05 ; * *: p<0.01$ 
versus $23.0(13.0-40.5) ; \mathrm{p}<0.001)$, and were taking fewer respiratory medications at baseline (median 3 (3-5) versus $4(3-5) ; \mathrm{p}=0.002)$. Asthma triggers differed, with exercise and reflux noted as significantly less common in patients who achieved control. Comorbidities were less prevalent in these patients; GORD (32\% versus $63 \%, \mathrm{p}<0.001)$, OSA ( $9 \%$ versus $30 \%, \mathrm{p}<0.001)$, vocal cord dysfunction $(3 \%$ versus $13 \%, \mathrm{p}=0.015)$, psychiatric disorders ( $17 \%$ versus $41 \%, \mathrm{p}<0.001)$, CVD ( $12 \%$ versus $31 \%, \mathrm{p}=0.002)$, diabetes $(7 \%$ versus $17 \%$, $\mathrm{p}=0.031$ ).

\section{Comparison of AMR patients with regulatory trial participants}

Compared to participants of the MENSA (Efficacy and Safety Study of Mepolizumab Adjunctive Therapy in Subjects With Severe Uncontrolled Refractory Asthma) and MUSCA (Efficacy and Safety Study of Mepolizumab Adjunctive Therapy in Participants With Severe Eosinophilic Asthma on Markers of Asthma Control) RCTs (supplementary table S9), AMR patients were older, with a longer duration of asthma, more maintenance OCS use, higher baseline ACQ-5 score and higher blood eosinophil levels. At the 6-month assessment, AMR patients reported a greater improvement in asthma control (ACQ-5) compared to MENSA/MUSCA participants.

\section{Discussion}

Mepolizumab is highly effective in treating severe refractory eosinophilic asthma in a real-world setting, where the treatment effect was as great, if not greater, than that reported in RCTs, despite the population having more severe asthma, being older and having a greater comorbidity profile. The beneficial effects observed included fewer severe asthma exacerbations, improved asthma symptom control, improved HRQoL and improved lung function. The drug was well tolerated, and the PBS selection criteria identified a group with a high individual response rate. Higher baseline blood eosinophils predicted a better response, and the drug was observed to be similarly effective in both atopic and non-atopic patients, as well as in those with early and late-onset asthma. We were able to identify a group of super-responders, who were predominantly women with high levels of T2 disease expression and few confounding comorbidities. These real-world data complement and extend the findings from highly controlled RCTs and provide further insight into response prediction when using mepolizumab in a clinical practice setting.

Predicting which patients will have the best response to mepolizumab (super-responders) is important, particularly as an individual patient with severe asthma may be eligible for several different add-on treatment options. Mepolizumab super-responders had shorter duration of asthma, less severe disease and had substantial treatment response. As such, appropriate patient phenotyping and targeting with mepolizumab therapy at an early time-point during the progression of disease; for example, as soon as asthma is identified as severe, may be advantageous in this context. Further characterisation of super-responders based on combined response criteria, including exacerbations and maintenance OCS use, will be beneficial.

The overall response rate to mepolizumab (based on PBS criteria for continuation) in the AMR was high at $86 \%$, and likely attributable to the effective phenotyping and subsequent targeted treatment according to the PBS initiation criteria. In agreement with the RCT findings, we also report enhanced clinical responses with increasing baseline blood eosinophils $[28,29]$. The median blood eosinophil level in AMR patients was 590 cells $\mu \mathrm{L}^{-1}$, which was significantly greater than that of the MENSA/MUSCA participants, and is a likely reason for the enhanced magnitude of clinical response observed in AMR patients, compared to the RCT participants, where baseline blood eosinophils levels of $\geqslant 150$ cells $\mu \mathrm{L}^{-1}$ were acceptable for eligibility $[9,11]$. AMR patients required blood eosinophil levels $\geqslant 300$ cells $\mu \mathrm{L}^{-1}$ during the 12 months prior to initiation (according to PBS-initiation criteria). However, the subsequent commencement of maintenance OCS in a proportion of these patients would be expected to result in a lower "true baseline" blood eosinophil level. As such, the reported relationship between baseline blood eosinophil level and the magnitude of response in this study is likely to be under-estimated in these patients. Future real-world evaluation of response in patients with blood eosinophil levels as low as 150 cells $\mu \mathrm{L}^{-1}$ will be informative, particularly when considering mepolizumab treatment for patients who have required ongoing maintenance OCS treatment and may not meet a higher blood eosinophil cut-off level.

In addition to elevated baseline blood eosinophil levels, AMR patients also had substantial baseline ACQ-5 scores (with an ACQ-5 score $\geqslant 2.0$ required for eligibility for initiation of mepolizumab therapy), which were higher than baseline scores reported in the RCTs, where there were no symptom score criteria for trial eligibility $[9,11]$. As such, the greater magnitude of ACQ-5 response observed in AMR patients relative to the RCTs is also likely attributable to the patients being more symptomatic at baseline.

Patients with late onset severe asthma are phenotypically distinct to those with earlier onset asthma, are generally non-atopic and often more severe [30,31]. Eosinophilia is present in both phenotypes, indicating that anti-IL5 targeted treatments may be of benefit regardless of age of onset. In order to evaluate response 
to mepolizumab treatment in severe eosinophilic asthma relative to age of onset, we chose a cut-off of $\geqslant 40$ years to identify late-onset asthma as described by others [32]. Consistent with other studies, early onset patients were predominantly atopic and had more allergic diseases, whilst the late onset group had more environmental exposures [33]. More than half of the late onset group were male, aligning with the recognised and difficult-to-treat adult-onset eosinophilic asthma phenotype [34, 35]. Despite the differing drivers of eosinophilic inflammation in these phenotypes, the baseline blood eosinophil levels were equivalent in AMR patients, regardless of age of onset, and we report similar clinical responses in each group. This is in agreement with a post hoc analysis of the COLUMBA study (Open-label Long Term Extension Safety Study of Mepolizumab in Asthmatic Subjects) [36]. Thus, targeted mepolizumab treatment in patients with severe eosinophilic asthma is likely to be of benefit regardless of the patient's age of onset phenotype.

Real-world observational studies have inherent limitations. As an observational post-marketing surveillance registry, patients in the AMR were not a randomised population, and the treatment trial was not controlled, and was therefore subject to bias and confounding factors. Response to treatment was assessed using the ACQ-5, a patient-reported symptom questionnaire, which may have led to response bias. However, it is a validated questionnaire for assessing treatment response and is used routinely in clinical practice [22]. The improvements in other outcomes such as reduction of severe exacerbations requiring hospitalisation/OCS treatment and improvements in lung function indicate that response bias was unlikely as these are objective measures of response. Another limitation identified in AMR was in the variability of comorbidity reporting, as this was a combination of physician diagnoses and patient reported comorbidity. However, the prevalence of comorbidities observed in the AMR were reflective of the rates expected in severe eosinophilic asthma. Nevertheless, despite their inherent limitations, real-world studies are gaining considerable acceptance when considering the ways in which these data complements and extends the findings of more tightly controlled studies in restricted populations.

\section{Conclusion}

The AMR reports that patients with severe uncontrolled eosinophilic asthma experience substantial disease burden and impact. We show that mepolizumab effectively reduces this burden, as demonstrated by a reduction in severe exacerbations and improvements in symptom control, health status and lung function. These findings complement the findings of RCTs and, in fact, suggest that when targeted appropriately in real-world clinical practice, patients can display superior clinical responses to mepolizumab. Further exploration of predictive factors and characterisation of mepolizumab responders in the real-world setting may be informative for future clinical management decisions.

Acknowledgements: We thank Siva P. Sivakumaran (Gold Coast University Hospital, Southport, Australia), Zinta Harrington (Liverpool Hospital, Liverpool, Australia) and Nicholas Harkness (Royal Hobart Hospital, Hobart, Australia) for contributing to the AMR.

Author contributions: All authors were involved in data interpretation and critical review of the manuscript. P.G. Gibson, E.S. Harvey, D. Langton, C. Katelaris, C.S. Farah, A. Gillman, J. Harrington, M. Hew, V. Kritikos, N. Radhakrishna, P. Bardin, M. Peters, P.N. Reynolds and J.W. Upham contributed to development of study concept and design. S. Stevens performed data analysis. E.S. Harvey, P.G. Gibson, D. Langton, C. Katelaris, C.S. Farah, A. Gillman, J. Harrington, M. Hew, V. Kritikos and N. Radhakrishna drafted the manuscript.

Conflict of interest: E.S. Harvey reports grants from GlaxoSmithKline that were paid to her employer, during the conduct of the study. D. Langton has received fees from GlaxoSmithKline for participation in severe asthma advisory boards. C. Katelaris reports grants from GlaxoSmithKline, during the conduct of the study; grants and personal fees for advisory board work and lectures from Sanofi, Novartis and CSL, personal fees from Seqirus and Takeda, outside the submitted work. S. Stevens has nothing to disclose. C.S. Farah reports personal fees from AstraZeneca, Boehringer Ingelheim, GlaxoSmithKline and Sanofi Genzyme, outside the submitted work. A. Gillman reports personal fees for advisory board work and education from GlaxoSmithKline, outside the submitted work. J. Harrington reports personal fees for education and advisory board work from AstraZeneca and GlaxoSmithKline, personal fees for education from Novartis, outside the submitted work. M. Hew reports grants and personal fees from AstraZeneca, GlaxoSmithKline and Novartis, personal fees from Sanofi, Teva and Seqirus, outside the submitted work; all paid to his institutional employer Alfred Health. V. Kritikos has nothing to disclose. N. Radhakrishna reports grants from Sanofi, outside the submitted work. P. Bardin reports per patient trial participation fees from Monash Lung and Sleep, during the conduct of the study; personal fees for advisory board work from Philip Bardin, outside the submitted work. M. Peters reports personal fees for advisory board work from Sanofi Genzyme, Novartis Pharmaceuticals and AstraZeneca, outside the submitted work. P.N. Reynolds reports grants from GlaxoSmithKline and AstraZeneca, during the conduct of the study. J.W. Upham reports grants and personal fees from AstraZeneca and GlaxoSmithKline, personal fees from Novartis and Boehringer Ingelheim, outside the submitted work. M. Baraket has nothing to disclose. S. Bowler reports personal fees for advisory board work from GlaxoSmithKline, outside the submitted work. J. Bowden reports personal fees for advisory board work from GlaxoSmithKline, AstraZeneca and Novartis, outside the submitted work. J. Chien reports personal fees from GlaxoSmithKline, outside the submitted work. L.P. Chung has nothing to disclose. C. Grainge reports personal fees from Boehringer Ingelheim, Roche Pharmaceuticals and GlaxoSmithKline, outside the submitted work. C. Jenkins reports personal fees for advisory board work, conducting meetings and developing educational 
content, and non-financial support from AstraZeneca, personal fees for advisory board work from Boehringer Ingleheim, grants and personal fees for advisory board work from GlaxoSmithKline, personal fees for advisory board work, facilitating symposia and developing educational content from Novartis, outside the submitted work. G.P. Katsoulotos has nothing to disclose. J. Lee reports personal fees for lectures from Boehringer Ingelheim and AstraZeneca, personal fees for consultancy from GlaxoSmithKline, outside the submitted work. V.M. McDonald reports grants and personal fees from GlaxoSmithKline, AstraZeneca and Menarini, outside the submitted work. H.K. Reddel reports grants from GlaxoSmithKline, during the conduct of the study; grants and personal fees for data monitoring committee work, advisory board work, providing independent medical education and consultancy from AstraZeneca, grants, personal fees for data monitoring committee work, advisory board work, providing independent medical education and consultancy and non-financial support (study medication) from GlaxoSmithKline, personal fees for data monitoring committee work from Merck, grants and personal fees for data monitoring committee work, advisory board work and providing independent medical education from Novartis, personal fees for providing independent medical education from Teva and Mundipharma, personal fees for advisory board work and providing independent medical education from Boehringer Ingelheim, personal fees for advisory board work from Sanofi Genzyme, outside the submitted work. J. Rimmer has nothing to disclose. P.A.B. Wark has nothing to disclose. P.G. Gibson reports grants from GlaxoSmithKline, during the conduct of the study; personal fees for lectures from AstraZeneca, GlaxoSmithKline and Novartis, grants from AstraZeneca and GlaxoSmithKline, outside the submitted work.

Support statement: The Australian Mepolizumab Registry is an investigator-initiated and managed study funded through the GlaxoSmithKline Investigator-Sponsored Studies program. Funding information for this article has been deposited with the Crossref Funder Registry.

\section{References}

1 McDonald VM, Gibson PG. Exacerbations of severe asthma. Clin Exp Allergy 2012; 42: 670-677.

2 Foster JM, McDonald VM, Guo M, et al. "I have lost in every facet of my life": the hidden burden of severe asthma. Eur Respir J 2017; 50: 1700765.

3 Lefebvre $\mathrm{P}$, Duh MS, Lafeuille $\mathrm{MH}$, et al. Acute and chronic systemic corticosteroid-related complications in patients with severe asthma. J Allergy Clin Immunol 2015; 136: 1488-1495.

4 Sweeney J, Patterson CC, Menzies-Gow A, et al. Comorbidity in severe asthma requiring systemic corticosteroid therapy: cross-sectional data from the Optimum Patient Care Research Database and the British Thoracic Difficult Asthma Registry. Thorax 2016; 71: 339-346.

5 Hiles SA, Harvey ES, McDonald VM, et al. Working while unwell: Workplace impairment in people with severe asthma. Clin Exp Allergy 2018; 48: 650-662.

6 Nair P, Pizzichini MM, Kjarsgaard M, et al. Mepolizumab for prednisone-dependent asthma with sputum eosinophilia. N Engl J Med 2009; 360: 985-993.

7 Haldar P, Brightling CE, Hargadon B, et al. Mepolizumab and exacerbations of refractory eosinophilic asthma. N Engl J Med 2009; 360: 973-984.

8 Pavord ID, Korn S, Howarth P, et al. Mepolizumab for severe eosinophilic asthma (DREAM): a multicentre, double-blind, placebo-controlled trial. Lancet 2012; 380: 651-659.

9 Ortega HG, Liu MC, Pavord ID, et al. Mepolizumab treatment in patients with severe eosinophilic asthma. $N$ Engl J Med 2014; 371: 1198-1207.

10 Bel EH, Wenzel SE, Thompson PJ, et al. Oral glucocorticoid-sparing effect of mepolizumab in eosinophilic asthma. N Engl J Med 2014; 371: 1189-1197.

11 Chupp GL, Bradford ES, Albers FC, et al. Efficacy of mepolizumab add-on therapy on health-related quality of life and markers of asthma control in severe eosinophilic asthma (MUSCA): a randomised, double-blind, placebo-controlled, parallel-group, multicentre, phase 3b trial. Lancet Respir Med 2017; 5: 390-400.

12 Brown T, Jones T, Gove K, et al. Randomised controlled trials in severe asthma: selection by phenotype or stereotype. Eur Respir J 2018; 52: 1801444.

13 Maltby S, Gibson PG, Powell H, et al. Omalizumab treatment response in a population with severe allergic asthma and overlapping COPD. Chest 2017; 151: 78-89.

14 Gibson PG, Reddel H, McDonald VM, et al. Effectiveness and response predictors of omalizumab in a severe allergic asthma population with a high prevalence of comorbidities: the Australian Xolair Registry. Intern Med J 2016; 46: 1054-1062.

15 Drick N, Seeliger B, Welte T, et al. Anti-IL-5 therapy in patients with severe eosinophilic asthma - clinical efficacy and possible criteria for treatment response. BMC Pulm Med 2018; 18: 119.

16 Lombardi C, Bagnasco D, Caruso C, et al. Analysis of the drop-out rate in patients receiving mepolizumab for severe asthma in real life. Pulm Pharmacol Ther 2019; 54: 87-89.

17 Pelaia C, Busceti MT, Solinas S, et al. Real-life evaluation of the clinical, functional, and hematological effects of mepolizumab in patients with severe eosinophilic asthma: Results of a single-centre observational study. Pulm Pharmacol Ther 2018; 53: 1-5.

18 Bagnasco D, Caminati M, Menzella F, et al. One year of mepolizumab. Efficacy and safety in real-life in Italy. Pulm Pharmacol Ther 2019; 58: 101836.

19 Pertzov B, Unterman A, Shtraichman O, et al. Efficacy and safety of mepolizumab in a real-world cohort of patients with severe eosinophilic asthma. J Asthma 2019: 1-6.

20 Caminati M, Cegolon L, Vianello A, et al. Mepolizumab for severe eosinophilic asthma: a real-world snapshot on clinical markers and timing of response. Expert Rev Respir Med 2019; 13: 1205-1212.

21 Numata T, Nakayama K, Utsumi H, et al. Efficacy of mepolizumab for patients with severe asthma and eosinophilic chronic rhinosinusitis. BMC Pulm Med 2019; 19: 176.

22 Juniper EF, Svensson K, Mork AC, et al. Measurement properties and interpretation of three shortened versions of the asthma control questionnaire. Respir Med 2005; 99: 553-558.

23 Nathan RA, Sorkness CA, Kosinski M, et al. Development of the asthma control test: a survey for assessing asthma control. J Allergy Clin Immunol 2004; 113: 59-65. 
24 Juniper EF, Buist AS, Cox FM, et al. Validation of a standardized version of the Asthma Quality of Life Questionnaire. Chest 1999; 115: 1265-1270.

25 Juniper EF, Bousquet J, Abetz L, et al. Identifying 'well-controlled' and 'not well-controlled' asthma using the Asthma Control Questionnaire. Respir Med 2006; 100: 616-621.

26 Harris PA, Taylor R, Thielke R, et al. Research electronic data capture (REDCap)-a metadata-driven methodology and workflow process for providing translational research informatics support. J Biomed Inform 2009; 42: 377-381.

27 Tay TR, Lee J, Radhakrishna N, et al. A Structured Approach to Specialist-referred Difficult Asthma Patients Improves Control of Comorbidities and Enhances Asthma Outcomes. J Allergy Clin Immunol Pract 2017; 5: 956-964

28 Ortega HG, Yancey SW, Mayer B, et al. Severe eosinophilic asthma treated with mepolizumab stratified by baseline eosinophil thresholds: a secondary analysis of the DREAM and MENSA studies. Lancet Respir Med 2016; 4: 549-556.

29 Albers FC, Licskai C, Chanez P, et al. Baseline blood eosinophil count as a predictor of treatment response to the licensed dose of mepolizumab in severe eosinophilic asthma. Respir Med 2019; 159: 105806.

30 Moore WC, Meyers DA, Wenzel SE, et al. Identification of asthma phenotypes using cluster analysis in the Severe Asthma Research Program. Am J Respir Crit Care Med 2010; 181: 315-323.

31 Schatz M, Hsu JW, Zeiger RS, et al. Phenotypes determined by cluster analysis in severe or difficult-to-treat asthma. J Allergy Clin Immunol 2014; 133: 1549-1556.

32 Brusselle G, Germinaro M, Weiss S, et al. Reslizumab in patients with inadequately controlled late-onset asthma and elevated blood eosinophils. Pulm Pharmacol Ther 2017; 43: 39-45.

33 Tan DJ, Walters EH, Perret JL, et al. Age-of-asthma onset as a determinant of different asthma phenotypes in adults: a systematic review and meta-analysis of the literature. Expert Rev Respir Med 2015; 9: 109-123.

34 Haldar P, Pavord ID, Shaw DE, et al. Cluster analysis and clinical asthma phenotypes. Am J Respir Crit Care Med 2008; $178: 218-224$

35 de Groot JC, Storm H, Amelink M, et al. Clinical profile of patients with adult-onset eosinophilic asthma. ERJ Open Res 2016; 2: 00100-2015.

36 Khatri S, Moore W, Gibson PG, et al. Assessment of the long-term safety of mepolizumab and durability of clinical response in patients with severe eosinophilic asthma. J Allergy Clin Immunol 2019; 143: 1742-1751. 